\title{
Scaling-up ICDS: Can Universalisation Address Persistent Malnutrition?
}

\author{
Rajani R. Ved*
}

\begin{abstract}
A$ countrywide initiative, the ICDS programme is India's primary response to addressing child malnutrition, but has had mixed success on the state of malnutrition in India. This article reviews the ICDS from the perspective of a scaling-up management framework and analyses aspects of design, advocacy, implementation and monitoring in the scaling-up of ICDS. Universalisation of ICDS with quality is well within the means of government and recent advocacy has resulted in increased funding; the scaling-up of ICDS is challenging. Successful scaling-up of ICDS requires the implementation of a multicomponent model, demanding a high level of quality and performance, coordination and convergence in the face of varying and limited management and technical capacity, poor governance environments, and little experience of engaging communities. Success in addressing these constraints is possible but attention to detail is critical and lessons should be adapted to suit local context.
\end{abstract}

\section{Introduction}

The Integrated Child Development Services (ICDS) is India's flagship programme for Early Childhood Development (ECD). Launched in 1975 , it is the country's major programme to address the needs of children under six, including malnutrition. Although substantial geographic scale has been achieved, there remains a significant percentage of the target 0-6 population who do not receive services, and there has been little improvement in malnutrition indicators. Despite the prima facie commitment of successive governments to ICDS, considerable pressure was required to be brought on the government from advocacy groups to achieve full scale with commensurate resources. The purpose of this article is to understand the reasons why the scaling-up of ICDS is at best mixed and to identify lessons that facilitate and pose challenges to successful scaling-up.

The precise issues the article examines are derived from a conceptual scaling-up management (SUM) framework developed by Management Systems International (Cooley and Kohl 2006). The framework, organised into three steps and ten tasks emphasises that successful scaling-up requires more than the adoption of a national and state policy and programme, and the allocation of increased financial resources. The SUM framework applied to the ICDS highlights several issues. First, it reviews the appropriateness of the intervention's design in light of the target population, underlying social context, and institutional capabilities of the organisation implementing the programme at large scale. Next, it emphasises the importance of advocacy, not only for formal adoption and financing, but for aligning the incentives of the entire implementation chain from politicians and senior bureaucrats to service providers and beneficiaries. Third, it focuses on the challenges of actual implementation at scale, especially a focus on whether the ICDS is afflicted by tendencies common to other large scale implementation, such as: little attention to quality; neglect of 'softer' components of the programme; and the dependency on the quality of local governance and capacity. Finally, it raises the importance of ongoing monitoring and feedback of large scale implementation, and the importance of maintaining political support for a long-term sustainable programme. 


\section{Description of the ICDS and its results}

The ICDS seeks to provide young children with an integrated package of services: supplementary nutrition (SNP), health services, and pre-school education (PSE). ICDS is primarily a central government-led initiative, but is implemented through the state governments. The ICDS centre in each habitation is referred to as an 'Anganwadi Centre' (AWC) and is managed by an 'Anganwadi Worker' (AWW). Supported by a helper, she is expected to carry out the functions of SNP, PSE and ensure convergence with the grassroots worker of the health system, to ensure health and nutrition services to children under six, pregnant and lactating mothers. The longstanding policy commitment to scaling-up ICDS countrywide was given a major impetus by a Supreme Court of India ruling in 2006, directing the Government to establish 1,400,000 AWCs (one for every settlement) with those for marginalised communities being a priority by December 2008. Begun as a small scale initiative in 33 blocks, ${ }^{1}$ the ICDS has now been scaled-up countrywide to 937,437 AWCs covering a population of 76.16 million for supplementary nutrition and about 30.81 million for pre-school education, ${ }^{2}$ of about 164 million children in the age group $0-6$ years.

Enrolment figures apart, the achievements of the ICDS to date are mixed. Data from the most recent National Family Health Survey (IIPS 2005-06) indicate that 46 per cent of India's children under three are undernourished, with about 53.2 per cent malnutrition among scheduled castes (SC) and 56 per cent among scheduled tribes (ST). Some 80 per cent of children aged 6-35 months are anaemic, and exclusive breast-feeding among children 0-5 months, is seen among 44 per cent. A total of 57 of every 1,000 children born, die before they reach the first year of life. These figures mask significant inter- and intra-state disparities.

Given India's size and diversity, the coverage, performance and impact of the ICDS programme at scale varies substantially, making it impossible to label the scaling-up of ICDS as successful or otherwise. However, the current state of malnutrition in the country reflects the limited impact of ICDS in its three decades of implementation. Recently, there has been a huge resurgence in the attention to ICDS, which could lead to improved performance and outcomes.

\section{Lessons from scaling-up ICDS 3.1 Design of the model}

The SUM framework emphasises the importance of an effective design. Certain types of intervention design can be scaled-up more easily than others, depending on whether it is technical or process interventions or whether it is a simple or a comprehensive model. Scalability also depends on the viability of the model when implemented across diverse social and institutional conditions, and its compatibility with the capabilities of the large scale implementing organisation. Many of these considerations do not appear to have been addressed in the ICDS model, perhaps helping to explain the mixed success of implementing this programme at scale.

A key design issue in ICDS is the absence of designing appropriate interventions for children in the various age cohorts between $0-6$ years, and the lack of attention to children below three years which is the time when nutrition interventions have the most effect. The ICDS, a centre-based intervention, whose programmatic focus was reduced to delivery of food to older children and mothers, needs substantial design modification to enable it to transform into an outreach model in order to communicate with mothers and caretakers to improve infant- and young child-feeding behaviours.

A second major design issue is the complexity of the ICDS model, consisting of eight elements, and encompassing a range of functions from changing norms and behaviours related to infant and young child-feeding, imparting pre-school education, to ensuring the provision of supplementary food to the target groups. ICDS also envisages convergence with health services, and the model is expected to be delivered at the peripheral level by a single worker often not well equipped for the task. ICDS also needs a well functioning procurement, supply and logistics system and effective supervision and monitoring.

Experience shows that a model with several components, some of which require substantial behaviour change, extensive outreach and the convergence of a number of government line departments, are less amenable to scaling-up than models with a few components, delivered vertically, emphasising technical solutions, and which do not rely on collaboration between various partners. 
A third design issue is the lack of consideration to location of the AWC, so that the marginalised and most vulnerable can be reached easily. Despite near universalisation, reach of the ICDS remains low and is accounted for by several factors inherent in the design. The AWC is often located in the centre of the village with accessibility to the village elite, resulting in exclusion of marginalised communities. The population-based norm of one centre per 1,000 also means that hamlets and smaller habitations do not have an AWC.

The fourth design issue to be considered for scalability is the ease with which the processes can be transferred to and adopted within large-scale systems. The ICDS was rolled out in various states with support from external donors and the central government. Excellent pilots and process innovations in ICDS were implemented in different corners of the country. Successes from small pilots, were well publicised, evaluation findings disseminated and advocated to policymakers as being a blueprint for the rest of the programme, across the country, often regardless of context and administrative environment. Since the focus of pilots and experiments was to demonstrate results, the role of mainstream institutions was often limited to implementation and participation in advisory committees. Limited attention was paid to advocating for a particular model with different states and providing technical support to integrate the lessons of the pilot into large-scale programmes.

For instance, the innovation of improved supervision and increased convergence between the health and nutrition departments in CARE's Rachana project was achieved by CARE appointed facilitators and liaison staff at district and block levels. This cadre of staff is not currently available within the government staff, posing a constraint when state and district programme managers want to scale-up the innovation. In contrast, innovations implemented by state governments, such as in the Tamil Nadu Integrated Nutrition Project (TINIP) resulted in better institutionalisation of processes and outcomes. The TINIP programme was implemented by the government itself and learning from the pilot incorporated into the larger state programme.

Finally, the initial testing of the model in the 33 blocks does not appear to have considered the ability of the service delivery system to handle these multiple requirements, resulting in gaps in the scaled-up effort. It appears that in scaling-up the interventions, in principle, fidelity to the model was considered more important than the capacity of the implementing organisations to deliver outcomes.

\subsection{Advocacy}

In the SUM framework the next key step in scaling-up is advocacy. Advocacy for scaling-up spans policy adoption, funding, implementation and sustainability. The review of these areas with regard to the ICDS model, show that until recently, advocacy for child health and rights was limited.

Policy adoption by the Central and State governments for ECD and the ICDS was relatively easy, but advocacy for adequate funding, implementation support and sustainable commitment appears to have been neglected. An analysis of parliamentary proceedings, between 2002 and 2006, show that only 3 per cent of questions raised in parliament pertained to children (Gitizens Initiative for the Rights of Children under Six 2006), reflecting low political commitment. Funding allocations and expenditures on ICDS likewise have been low, until recently. From 1975 to 1992, spending on ICDS was about Rs. 700 million. In contrast, the WCD department's projection for the 11 th Five-year Plan (2007-2012) is for US $\$ 18$ billion (GoI 2007).

This represents a fairly dramatic increase in funding and reflects recent advocacy for the issue of child health. Over the last five years, there has been a spate of surveys and reports drawing attention to India's failure to reduce malnutrition among children. This has led to significant political attention, including civil society activism. Part of this advocacy has its genesis in the Right-to-Food Case, filed by the People's Union for Civil Liberties vs. Union of India in 2001. The Supreme Court of India took the view that denial of the 'right-to-food' amounts to the denial of the fundamental 'right to life and personal liberty' and saw the ICDS as central to safeguarding the right to food of young children. Over the past few years the Supreme Court $^{3}$ has passed several progressive rulings forcing the central and state governments to ensure expanded coverage of the ICDS. During 
the same period, even while India committed to meeting the Millennium Development Goals, several large scale surveys such as the National Family Health Survey drew attention to the persistence and even deterioration of malnutrition among India's children. This combination of factors led to heightened advocacy for the issue of childhood malnutrition, prompting increased media and public attention, and increased political commitment, all of which are critical to effective scaling-up.

The SUM framework emphasises that advocacy is needed beyond obtaining additional resources and attention. In fact, advocacy during the implementation phase of scaling-up and even after scaling-up has been achieved is essential to ensure that commitment to ICDS does not falter.

\subsection{Implementation}

The SUM framework identifies as the third step, attention to implementation at scale with a focus on: governance environment, human resources, capacity building, infrastructure, logistics and supply chains, incentives and organisational culture. We apply this to the ICDS model, and show that this is probably the area with the most significant set of breakdowns, with the quality of governance being an important factor in determining implementation effectiveness.

The first lesson here is the importance of governance conditions in implementation. This can be seen by contrasting the scale-up of the food components of the programme in a well governed state like Tamil Nadu, where the political compulsion of food distribution has been used to good effect. The convergence of strong political will (constant over three decades, and immune to the vagaries of changing political parties in power), high levels of community awareness, effective people's participation in the programme, and strong implementation capacity, resulted in a well performing ICDS at scale, which has now incorporated other elements besides nutrition.

Given that the political appeal of food distribution programme is universal, why has this not happened in other states? The answer lies perhaps in the structure of governance and social structures in Tamil Nadu, the fact that caste barriers in ICDS are purposefully approached and mitigated to some extent through community action, the potential of collectives realised through active involvement of self-help groups and effective convergence at the field level is ensured on account of a wellfunctioning public health system.

Poor governance impacts functioning of scaledup programmes, regardless of evidence of impact. Thus the multi-component model of ICDS with a high reliance on good governance and accountability mechanisms, which was well implemented in a state like Tamil Nadu, has been advocated for states like Uttar Pradesh and Bihar with little consideration of political structures, programmatic readiness and environment. The sixth report (Saxena and Mander 2005) of the Commissioners to the Supreme Court highlights corruption in food procurement, distribution, in appointments of anganwadi workers, and general indifference to ICDS functioning in those states.

The second issue with scaling-up ICDS relates to the varying implementation capacity across states. Little appears to have been done with either modifying and strengthening organisations to undertake the implementation of a scaled-up programme or adapting the intervention to suit the needs of the state.

Although in theory the universalisation or scaling-up of ICDS advocates decentralisation and local adaptation, in practice, given the limited implementation capacity of several states, little local adaption was attempted. The states in which prevalence of malnutrition is high are also the states where both state and central government allocation of funds for ICDS is low (Gragnolati et al. 2005). Official apathy and public inertia often feed upon each other, mutually reinforcing the status quo. This affects all aspects of the programme.

The third issue that has hampered the scaling-up of ICDS is the lack of attention paid to providing the AWW with the capacity and skill to deliver services. Scaling-up requires both an expansion of human resources and a well-developed capacity building strategy to enable requisite skills and competencies. Her performance is constrained by poor quality of training and the pressure of a large and diverse workload. The ICDS has over a million workers and helpers to be trained. Capacity building is currently 
through institutions whose mandate is solely training and whose trainers have no real understanding of programme implementation issues, thus divorcing capacity building from implementation. Training of AWW is delivered by trainers who have little inkling of field realities. ${ }^{4}$ Despite several innovations in training such as the World Bank funded Udisha project, there is a gap between the training programme and the reality the worker encounters in the field, given that the training curriculum, methods and modules are centrally designed. Ongoing mentoring in addition to formal preand in-service training is critical in large scale programmes to ensure that skills do not get eroded. Currently ICDS supervisors (who could serve as field mentors) are more focused on enrolment and attendance data, and salaries rather than on reviewing processes and performance.

Neither the anganwadi worker who is the lynch pin of the ICDS programme, supervisors or programme managers, have incentives for high performance. On the contrary, the FOCUS report cites that the main concerns of the anganwadi worker includes 'inadequate infrastructure; lack of training; low and irregular salaries; excessive work overload; lack of community support; and intimidation or extortion from the supervisors'. Motivating the anganwadi worker through monetary or in kind incentives to deliver high quality services may have yielded better returns.

Finally, implementation of the scaled-up programme requires strong programme management support units at state and district levels, including supervision and mentoring of grass roots staff to undertake a plethora of interventions. Rarely do programme reviews comment on the management of the ICDS, except to highlight staff vacancies at district and lower levels. There have been few changes in the composition, structure and capacity of state and district programme management units in several years, regardless of the roll out of the programme and the need for increased human resources.

\subsection{Monitoring and feedback}

The SUM framework emphasises that even after the intervention has been scaled-up, there is a need for continuing performance monitoring and feedback to sustain the intervention at scale.
Although several small and large scale studies were conducted in the past three decades, the focus of the studies appears to be on assessing programme inputs, coverage, enrolment and infrastructure, rather than outcomes. A significant exception to this is the FOCUS report, which examined the various facets of the ICDS programme and succeeded in highlighting the strengths and limitations of ICDS. Even though this was not an impact evaluation, its conclusions and recommendations provide the impetus to improve implementation.

There is little evidence from existing literature that ICDS scaling-up envisioned a systematic strategy based on a review and understanding of the results of the pilot initiative, or thereafter. The scaling-up of ICDS, focused on expanding geographic coverage and followed the same design as the pilot model, even though programmatic reviews, despite constraints of quality and validity, highlighted that implementation was skewed with some components receiving greater attention than others, affecting impact.

Performance assessment for improved implementation can only take place when there are well established routine monitoring and supervisory systems. Routine monitoring systems in the ICDS programme require substantial overhaul. Presently, the anganwadi worker is taxed with filling in about $10-25$ registers, depending on the state in which she works. Despite this however, there is little by way of data to demonstrate the actual performance of the ICDS vis-à-vis the various parameters. Continuous monitoring of programme implementation, effective supervisory systems, setting up feedback loops and performance indicators, are critical at all stages of scaling-up. This is one area which was not integrated into the ICDS even in its early days, resulting in an ineffective $\mathrm{M} \& \mathrm{E}$ system, limited in its ability to measure outcomes.

Programmes operating at scale need to assimilate new knowledge, generated internally as well as new research, and have the flexibility to adapt. The current nutrition supplement of 300 calories was based on research in the 1960 s and 1970 s, which recommended a cereal-pulse mix (UNICEF 2007). Recent studies show that the calorie gap is much higher among children 
3-6 years. The cereal pulse recommendation has been converted to a cereal-based supplement, thus reducing the intake of most nutrients. Learning from new research and examining the effects of existing programme components are only possible in learning organisations with carefully designed monitoring systems.

\section{Conclusions}

Despite several shortcomings, it is clear that there is both a significant need and a tremendous opportunity to improve the effectiveness of ICDS functioning at scale.

The past few years have seen substantial civil society and judicial activism which in turn has generated greater public interest and political commitment to the cause of children. Factors that are likely to positively influence the outcomes of the ICDS in the future include: The Right-to-Food Movement, the Right to Information Act, and intensive monitoring by the Supreme Court appointed Commissioners to monitor 'Universalisation with Quality'.

The portents of high levels of sustained funding to the ICDS programme bode well, signalling a policy commitment as never before. The Country's XI plan allocation (2007-12) for ICDS is substantially higher than at any time in the past. Several state governments have piloted innovations in the implementation of ICDS, resulting in improved nutrition outcomes for mothers and children, with high potential of replication. The design of ICDS IV appears promising. Among the large number of reforms that it envisions through a Mission mode approach, it includes, inter alia, provision for a two worker Anganwadi Centre, ensuring division of labour and skills enabling effective delivery of all components, decentralised planning, provision of flexible funds, commitment to inclusion and equity, and responsive monitoring and information systems.

Lessons from scaling-up ICDS highlight the fact that action is required on several fronts.

Sustained advocacy to keep the spotlight on ICDS functioning is critical. Scaling-up requires decentralised management and local adaptation. The multiple components of ICDS need a high level of coordinated management and performance monitoring. Programmes operating at scale need to assimilate new knowledge and research generated internally, and have the flexibility to adapt. ICDS scaling-up faltered where convergence was required with different stakeholders such as the departments of local governance, health and education. ICDS will need to address issues of equity through design modification and implementation, even while it is attempting large scale reach.

To deliver on the promise and potential of the ICDS IV, the ICDS needs sustained political and financial commitment, leadership at all levels, community ownership and a substantial modification in implementation modalities. The complexity of the design of the ICDS and the uniformity of design across the country with varying governance, administrative, and implementation challenges advocates the need for states to attempt simplified models within the ICDS and phasing in the more complex issues over a specified time period. The issue of limited management capacity to undertake programmes at scale has been addressed to some extent in the health and education sector through capacity building, decentralised planning and implementation, incentives and systemic changes to enforce accountability.

Lessons from India's flagship National Rural Health Mission (GoI 2008), which includes measures to address low implementation capacity, lack of community mobilisation, poor training and capacity building, and improving MIS demonstrate that support from the central government for effective planning within decentralised systems, sustained oversight, frequent monitoring and sharing of lessons across states have contributed to improving health systems.

Scaling-up requires strategic planning that includes a sound understanding of the political and governance environment, and designing organisational structures and institutional mechanisms to deliver at scale which are context specific. Scaling-up plans for state and district level need to include specific know-how and should draw upon local experiences and local resources, including community. Finally advocacy efforts need to continue to ensure that there is no backsliding and that ICDS is insulated from political manipulation and vested interests that are inimical to its functioning. 


\section{Notes}

* Disclaimer: The opinions, conclusions and findings in this article do not necessarily represent the views of MSI or the Packard Foundation. Support for background research required for the article was provided by the Packard Foundation.

1 A block is a unit of planning that covers a population of about 100,000. Roughly 100 Anganwadi Centres are expected to be operational in one block.

\section{References}

Citizens Initiative for the Rights of Children under Six (2006) FOCUS on Children under Six, New Delhi: Citizens Initiative for the Rights of Children under Six

Cooley, Larry and Kohl, Richard (2006) Scalingup-from Vision to Large Scale Change: A Management Framework for Practitioners, March, Washington DC: Management Systems International

GoI (2008) Second Common Review Mission, Nov-Dec 2008, Government of India: National Rural Health Mission, Ministry of Health and Family Welfare

GoI (2007) ICDS IV, Project Concept Note (Revised September), Government of India: Project Management Unit, Ministry of Women and Child Development

Gragnolati, M.; Shekar, M.; Das Gupta, M.; Bredenkamp, C. and Yi-Kyoung Lee (2005)
2 As of September 2007, Annual Report of Ministry of Women and Child Development 2007-08.

3 www.righttofoodmovement.org

4 Reforming ICDS for Greater Impact: Issues and Options for ICDS in the 11th Five-Year Plan, CARE 2007, unpublished document.

India's Undernourished Children: A Call for Reform and Action, Health, Nutrition and Population (HNP) Discussion Paper, Washington DC: World Bank

International Institute of Population Sciences (2005-06), National Family Health Survey 3, Government of India: Ministry of Health and Family Welfare

Saxena, N.C. and Mander, Harsh (2005) Sixth Report of the Commissioners, New Delhi: Office of the Commissioners of the Supreme Court

UNICEF: Working Group on Children Under Six (2007) 'Strategies for Children Under Six', Economic and Political Weekly, 29 December, http://unicef.globalstudy.googlepages.com/ EPWARTICLEStrategiesforChildrenunder.pdf (accessed 22 April 2009) 